\title{
Realtime Characterization of In Situ Molecular Alignment during the Saponification Process of Polyvinyl Pivalate
}

\author{
Byoung Chul Kıм${ }^{\dagger}$, Won Seok Lyoo*, and Wan Shik HA** \\ Division of Chemical Engineering, Hanyang University, \\ 17 Haingdang, Seongdong, Seoul 133-791, Korea \\ ${ }^{*}$ School of Textiles, Yeungnam University, \\ Kyungsangbuk-do 712-749, Korea \\ **Department of Fiber and Polymer Engineering, Seoul National \\ University, Seoul 151-742, Korea
}

(Received August 16, 1999)

\begin{abstract}
The spontaneous fiber formation process during saponification of poly(vinyl pivalate) (PVPi) with syndiotactic diad content 63 to $65 \%$ to poly(vinyl alcohol) (PVA) in the presence of water was in situ examined. The variation of the revolution per minute(rpm) of a torque apparatus under a constant torque was measured for the spontaneous fibrillation system, and the actual fibrillation system was visualized in the realtime scale by in-line photographing. The in situ fibrillation mechanism was accounted for by relating the variation of the rpm with proceeding the saponification reaction to the change of solution morphology with saponification. At the constant torque level, the rpm changed with proceeding the saponification reaction in a complicated way. Up to the saponification time $80 \mathrm{~s}$, the rpm was slightly increased with saponification, then decreased to the saponification time $400 \mathrm{~s}$. From the saponification time $400 \mathrm{~s}$, however, the rpm was abruptly increased. Then, the rpm was levelled off after the saponification time $700 \mathrm{~s}$. Comparison of the rotational speed variation with the morphology change revealed that phase separation began from the degree of saponification $72.8 \%$, and a clear fibrillar structure appeared from the degree of saponification $91.6 \%$.

KEY WORDS Polyvinylpivalate / Polyvinylalcohol / Saponification / Visualization / In Situ Fibrillation
\end{abstract}

In the case of vinyl polymers with asymmetrical substituents, the conformation of polymer molecules is affected by the tacticity. Due to the rotational isomerism on the asymmetric carbon center, the vinyl polymers can take three different conformations depending on the spatial arrangement of the pendant groups to the main chain backbone ; atactic, isotactic, and syndiotactic. Tacticity has a significant effect on the physical properties of the polymer. Particularly with vinyl polymers containing polar pendant groups such as poly(vinyl alcohol) (PVA), the effect of tacticity would be much more noticeable because the conformation determines the extent of the intermolecular interactions caused by the polar pendant groups.

PVA is prepared by alkali hydrolysis of poly(vinyl ester) precursors such as polyvinyl acetate (PVAc) and poly(vinyl pivalate) (PVPi). Due to steric hindrance of bulky pendant group PVPi gives higher syndiotactic diad content than PVAc. On the whole, the latter has the syndiotactic content 52 to $53 \%$ while the former has the syndiotactic content 63 to $65 \%$. The saponification of PVPi solution in tetrahydrofuran (THF) by the solution of potassium hydroxide in the mixture of methanol and water leads to the in situ formation of PVA fiber by the spontaneous alignment of polymer molecules. ${ }^{1}$ On the other hand, PVAc does not produce any fibrillation in the same saponification process. ${ }^{1}$

The PVA fiber thus prepared has a natural pulplike structure in which bundles of microfibers are laminarlyassociated within the fiber. The laminarly-associated fiber structure is encountered largely in the naturally occurring cellulose fibers such as jute and flax. Recently, however, it was reported that the laminar-structured fibers were also obtained by specially devised nonspinning processes such as growth-packing synthesis of poly $(p$-phenylene terephthalamide) and in situ fibrillating extrusion of supercooled hydrated acrylic melts. ${ }^{2}$

We attempted to account for the in situ spontaneous fibrillation mechanism in the saponification process of syndiotactic-rich PVPi. ${ }^{3,4}$ The rheological characterization of the solutions of PVA with different syndiotactic contents revealed that the tacticity of polymers had a critical effect on the rheological properties of the PVA solutions in dimethyl sulfoxide (DMSO) ${ }^{3}$ PVA prepared from PVAc (the syndiotactic diad content 52 to $53 \%$ ) shows rheological properties typical of most flexiblechain polymers. On the other hand, PVA prepared from PVPi (the syndiotactic diad content 63 to $64 \%$ ) exhibits rheological responses frequently encountered in the liquid crystalline polymers, particularly in the nematic phase. The syndiotactic content of the polymer had a profound effect on the rheo-optical properties as well. ${ }^{2}$ We estimated the mechanism of the spontaneous fibrillation by examining changes in the rheological properties of PVPi solutions in DMSO at different degrees of saponification. ${ }^{4}$ This result coincide with the result of Yamaura et al ${ }^{5}$ They reported that syndiotacticity-rich PVA gives rise to fibrillation by shear-induced crystallization from aqueous solutions.

All these rheological efforts, however, could not give a satisfactory explanation on the mechanism of the in situ fiber formation. It offered only an indirect clue to the fiber formation mechanism because the PVA solutions for rheological measurement could not contain water. As

\footnotetext{
${ }^{\dagger}$ To whom all correspondence should be addressed (e-mail : bckim@email. hanyang. ac. kr).
} 
might be imagined, water brings about a serious phase separation and gelation during rheological measurement. In reality, however, water plays a decisive role in the in situ fibrillation process. This study traced the actual in situ fibrillation process during the saponification of PVPi by on-line measurement of the rotational speed change under a constant torque and realtime visualization of the in situ fibrillation process.

\section{EXPERIMENTAL}

\section{Preparation and Saponification of Syndiotactic-Rich $P V P i$}

In the in situ fibrillation of PVPi during saponification, a high molecular weight syndiotactic-rich precursor polymer with little branched structures is required. For this purpose, the precursor polymer was prepared by low temperature ultraviolet-initiated bulk-polymerization of vinyl pivalate with a bulky pendant-group. ${ }^{1}$

The PVPi so prepared was saponified to PVA as follows. $4 \mathrm{~g}$ PVPi was dissolved in $200 \mathrm{~mL}$ THF and $5 \mathrm{~g}$ potassium hydroxide was dissolved in $20 \mathrm{~mL}$ of THF/water (90/10 by vol.). To the PVPi solution the potassium hydroxide solution was added drop by drop with vigorous stirring at $60^{\circ} \mathrm{C}$. The solid reactant was beaten mechanically. Filtering and washing the beaten product with methyl alcohol yielded a bright yellow PVA fiber.

\section{Measurement of Molecular Parameters}

To measure the molecular weight of polymer, PVA was reacetylated with anhydrous acetic acid and acetic anhydride in the presence of pyridine under nitrogen bleeding at $100^{\circ} \mathrm{C}$ for $72 \mathrm{~h}$. The reacetylated PVAc was dissolved in benzene $\left(0.5 \mathrm{~g} \mathrm{dL}^{-1}\right)$, and the molecular weight of the polymer was determined by eq $1^{6}$

$$
[\eta]=8.91 \times 10^{-3}\left[P_{n}\right]^{0.62}
$$

in which, $[\eta]$ is the intrinsic viscosity at $30^{\circ} \mathrm{C}$ and $P_{n}$ is the number-average degree of polymerization.

The degree of saponification was determined by weight loss after saponification and by the ratio of $t$ butyl and methylene proton peaks in the ${ }^{1} \mathrm{H}$ NMR spectrum. The syndiotactic diad content of PVA was determined by $300 \mathrm{MHz}{ }^{1} \mathrm{H}$ NMR spectrometer (Varian Sun Unity) using DMSO- $d_{6}$ as a solvent, based on the ratio of the components of the hydroxy proton triplet at $4.1-4.7$ ppm. ${ }^{6}$ PVA with the syndiotactic diad content of 63 to $65 \%$ was obtained from PVPi, which is comparable with PVA with the syndiotactic diad content of 52 to $53 \%$ obtained from PVAc.

\section{Measurement of the In Situ Fibrillation Process}

The in situ fibrillation process during saponification of PVPi was assessed by measuring the rpm with saponification time. The in situ fibrillating morphology was inline observed by video microscope (Scalar VMS-3000) equipped with a Sony color video printer. To obtain good reproducibility a cylindrical saponification apparatus with simple-shaped symmetric blades was used. The probe of the microscope was installed in the wall of the apparatus. The microstructure of the in situ formed PVA fiber was observed with a scanning electron microscope
(SEM ; Hitachi S-510).

\section{RESULTS}

The rpm at a given constant torque was measured for the PVPi system under saponification by on-line monitoring rotational speed apparatus, and variation of the rpm with saponification time (a measure of the degree of saponification) is shown in Figure 1. With proceeding the saponification reaction the rpm varies in a very complicated manner. Up to the saponification time $80 \mathrm{~s}$, the $\mathrm{rpm}$ is slightly increased with saponification. It is then decreased till the saponification time $400 \mathrm{~s}$. However, the rpm is abruptly increased again with saponification, then levelled off after the saponification time $700 \mathrm{~s}$.

In order to visualize the in situ fibrillation process during the saponification of syndiotacticity-rich PVPi, the change of the solution morphology in the PVPi solution under saponification was traced. Figure 2 shows a series of microscopic photographs tracing the morphological change in the actual saponifying PVPi system. The photographs exhibit a gradual change of the solution morphology with proceeding the saponification reaction. The solution gets heterogeneous with saponification. A fibrillar structure begins to appear from the degree of saponification $72.8 \%$, and a fiber shape is observed from the degree of saponification $91.6 \%$. Finally a well-oriented fiber structure is obtained at the degree of saponification $98.5 \%$.

\section{DISCUSSION}

The saponification of PVPi by potassium hydroxide in the mixture of THF and water yields a natural pulp-like PVA fiber in which numerous bundles of highly oriented and crystalline microfibers are laminarly associated.1,3 As previously reported, of two precursor polymers of PVA, PVAc, and PVPi, the in situ fiber formation during saponification is observed only for the syndiotactic-rich

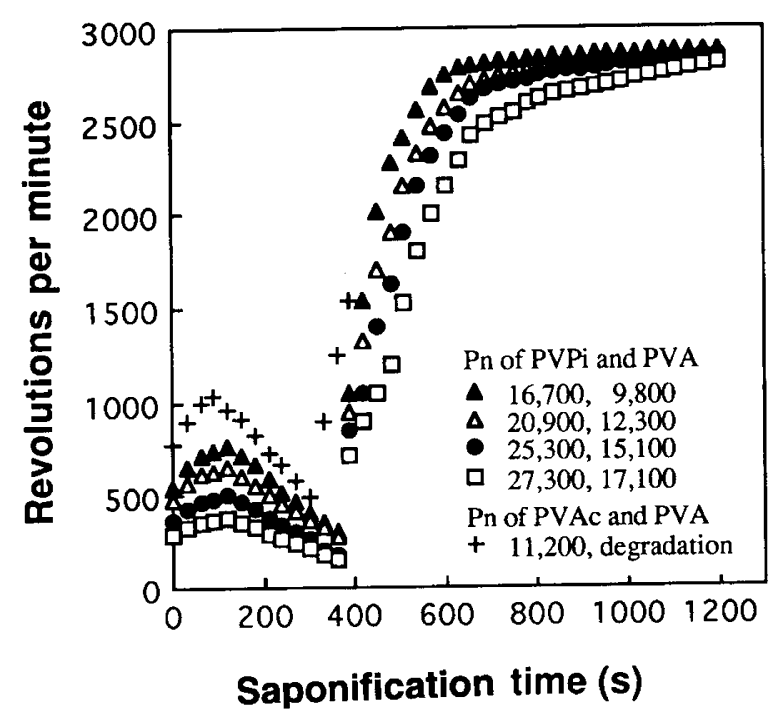

Figure 1. Variation of rpm at a constant torque with saponification time during saponification of syndiotacticity-rich (PVPi)s with different molecular weights (the molecular weight of PVA represents the resultant value after having saponified the corresponding PVPi). 


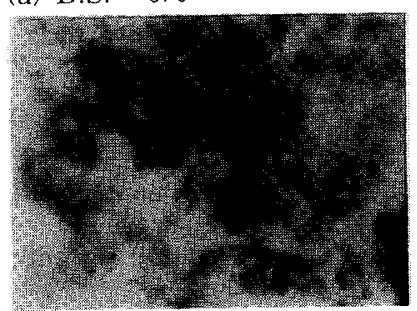

(c) D.S. : $72.8 \%$

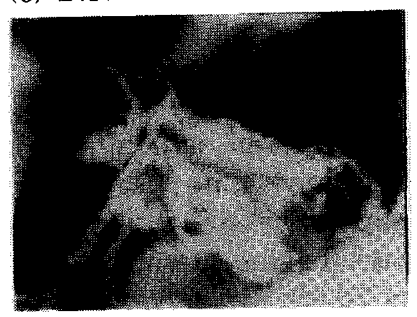

(e) D.S. : $98.5 \%$

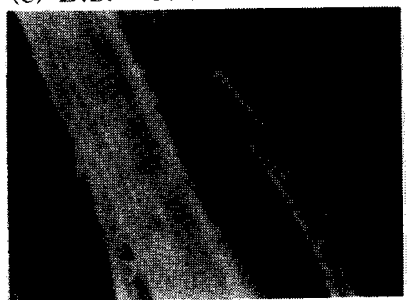

(a) D.S. : $0 \%$ (b) D.S. : $13.1 \%$

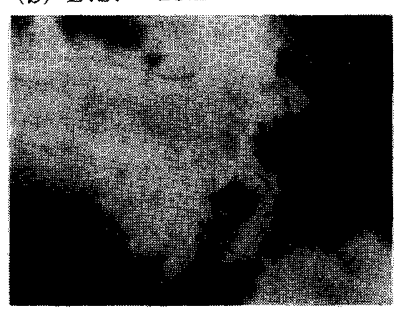

(d) D.S. : $91.6 \%$

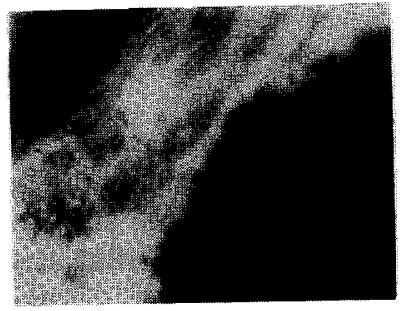

Figure 2. Change in phase morphology with proceeding the saponification reaction in saponification of syndiotacticity-rich PVPi with potassium hydroxide, showing the realtime process of the in situ formation of PVA fiber.

PVPi (the syndiotactic diad content 63 to $65 \%$ ) but the saponification of PVAc with the syndiotactic diad content $52-53 \%$ does not yield any fibrillar products. This suggests that the tacticity of the precursor polymers has a decisive effect on the in situ fiber formation. ${ }^{3,4}$

Syndiotacticity-rich PVA offers more favorable conditions for the intermolecular interactions by hydroxyl groups because the larger number of the hydroxyl groups are regularly arranged as schematically illustrated in Figure 3. Hence the strong intermolecular attraction would result in formation of some physically associated structures. In fact, the $2 \mathrm{wt} \%$ solutions of the syndiotacticity-rich PVA obtained from PVPi in DMSO exhibited the rheological properties different from ordinary polymer solutions. The solutions give slopes much less than 2 on the logarithmic plot of storage modulus against loss modulus and yield behavior on the viscosity curve. ${ }^{3}$ These rheological characteristic features are indicative of heterogeneous systems, frequently encountered in the anisotropic phase of the liquid crystalline polymers and inorganic-filled polymer systems. Interestingly, applying the slightest shear to the $2 \mathrm{wt} \%$ solutions of PVA with syndiotactic diad contents 63 to $65 \%$ produces fibrillated structures like the anisotropic phase of liquid crystalline polymers. ${ }^{4}$

In Figure 1 the rpm measured at a constant torque can be regarded as a measure of the reciprocal of shear viscosity although the apparatus does not give a perfectly uniform shear field throughout the solution and

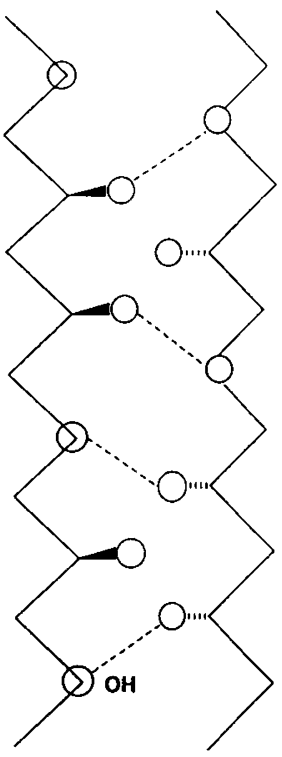

(a)

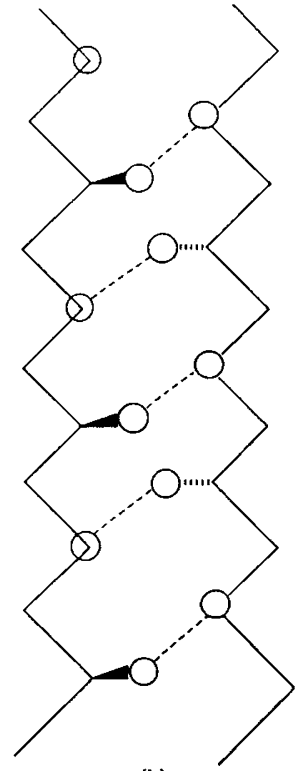

(b)
Figure 3. Schematic representation of molecular conformations of (a) pure atactic and (b) pure syndiotactic (PVA)s: The dotted line indicates hydrogen bond $(O$; hydroxyl group, $\varnothing$; downward, and $\alpha$; upward).

the flow accompanying chemical saponification reaction is not exactly laminar. For convenience of understanding, however, the saponification process may be accounted for on this assumption. The rpm data with saponification exhibited a good reproducibility when the cylindrical saponification apparatus equipped with simpleshaped symmetric blades was used. The slight decrease in viscosity up to the saponification time $80 \mathrm{~s}$ is due to mixing of saponifier and PVPi. The increase of viscosity till the saponification time $400 \mathrm{~s}$ is ascribable to gelation by the incorporated potassium hydroxide solution.

The extent of intermolecular interactions is increased as the degree of saponification is increased. Thus, increasing the degree of saponification offers more favorable condition for the phase separation of polymers by the physical aggregation of polymer molecules in the solution. In fact, the degree of shear thinning was increased with increasing the degree of saponification. ${ }^{4}$ The higher degree of shear-thinning at the higher degree of saponification indicates that the polymer chains form stronger gel structures as the saponification reaction is proceeded. This indicates that increasing the degree of saponification makes shear-induced molecular orientation easier.

From the late stage of the saponification process at which lots of pivalate groups are hydrolyzed to hydroxyl groups, water plays a significant role in the physical association of polymer molecules through hydrogen bonding. The water molecules seem to promote lateral arrangement of polymer molecules by physically binding the hydroxyl groups of different polymer chains. Thus, the water bridging between hydroxyl groups may lead to three-dimensionally ordered gel structures. Hence the solution viscosity exhibited maximum in the vicinity of the saponification time $400 \mathrm{~s}$.

At the final stage of the saponification process, the intermolecular attraction by hydrogen bonding may be 


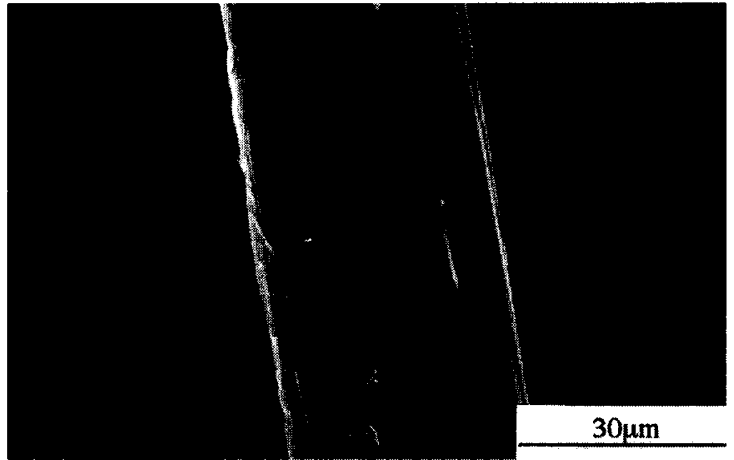

Figure 4. Microstructures of the in situ formed PVA fiber (DP= 12300).

strong enough to yield hydrogel structure, which may bring about phase-separation with the aid of shear. With further increase of the degree of saponification shearing will change the gel structure to the fibrillar structure because the gel comes to possess a physical structure strong enough to retain a permanent deformation by shear. An abrupt decrease in viscosity from the saponification time between 400 and $700 \mathrm{~s}$ reflects this shearinduced structural transformation. Thus, the decrease of viscosity is attributable to the orientation of the fibrillar product formed by phase separation to the shear direction.

PVA is a limiting case of saponification, implying almost $100 \%$ saponification. Shearing the syndiotacticityrich PVA solution readily fibrillate the polymer molecules to the shear direction between glass plates. ${ }^{4}$ In the case of PVA, a transition of polymer molecules from flexible to rigid conformation seems to take place if the content of syndiotacticity exceeds a critical value. This is conceived from the fact that the solutions of (PVA) $s$ with the syndiotactic diad content 52 and $64 \%$ in DMSO exhibit completely different rheological responses, ${ }^{3,4}$ typical of homogeneous polymer solutions and heterogeneous polymer systems, respectively. The critical content of the syndiotacticity for the conformational transition seemed to be $54.3 \%$ because clear fibrillation during saponification was obtained from the syndiotacticity content. ${ }^{8}$ The rheological responses suggest that such a conformational transition may occur during the saponification of syndiotacticity-rich PVPi if the degree of saponification exceeds the critical value, $54.3 \%$. The necessity of water for the in situ saponification process of PVPi clearly indicates that hydrogen bonding plays a key role in the in situ fiber formation process.

The viscosity of the PVPi solution system under saponification remains almost invariant after the saponification time $700 \mathrm{~s}$. This may suggest that the fiber formation process has been completed at that time. That is, since almost all the PVA produced by saponification of PVPi is already separated out from the solvent as very thin microfibers there is only a little change in solution composition with further saponification. The low viscosity of the resultant system is obliged to the low concentration of the solid polymer content in the system, less than $2 \mathrm{wt} \%$.

The change of the solution morphology during the saponification of syndiotacticity-rich PVPi in Figure 4
Table I. Relation between saponification time, degree of saponification, and morphological change in the real saponification process of syndiotacticity-rich PVPi with potassium hydroxide

\begin{tabular}{crrl}
\hline $\begin{array}{l}\text { Saponification Saponification } \\
\text { stage }\end{array}$ & $\begin{array}{l}\text { Degree of } \\
\text { time/s }\end{array}$ & $\begin{array}{l}\text { saponifica- } \\
\text { tion/\% }\end{array}$ & Change in phase morphology \\
\hline 1 & $0-120$ & $<15$ & $\begin{array}{l}\text { Mixing PVPi solution with } \\
\text { saponification agent }\end{array}$ \\
2 & $120-360$ & $15-80$ & $\begin{array}{l}\text { Gelation of the saponify- } \\
\text { ing system }\end{array}$ \\
3 & $360-480$ & $80-90$ & $\begin{array}{l}\text { Abrupt collapse of gel structure } \\
\text { by breakdown of solvent bridges } \\
4\end{array}$ \\
$480-1200$ & $90-99+$ & In situ fibrillation
\end{tabular}

shows that increasing the degree of saponification makes the solution more heterogeneous, and fibrillation results at the final stage of saponification. It should be noticed that a fibrillar structure begins to appear from the degree of saponification of $72.8 \%$ which corresponds to the saponification time $400 \mathrm{~s}$. A clear fiber shape is observed from the degree of saponification $91.6 \%$ which is equivalent to the saponification time $700 \mathrm{~s}$. A welloriented fiber structure is obtained at the degree of saponification $98.5 \%$, which means the completion of the fiber formation process. These realtime-scale photographs ascertain that the rheological reasoning based on the rpm measured at a constant torque is creditable. Thus, relating the change in the solution morphology to the variation of the rpm enables us to assess the mechanism of the spontaneous in situ fiber formation during saponification of syndiotacticity-rich PVPi. The experimental results verifies that the morphological change with saponification well coincides with the rpm variation with saponification as compared in Table I .

As well recognized, fibers should possess rigid domains or crystallites to have good mechanical properties because the oriented molecules are thermodynamically unstable and should be stabilized by pseudocrosslinking structures. Although there is not a stretching step nor an annealing step during the fiber formation process, Xray analysis shows that the in situ fibrillated PVA fiber has the high degrees of orientation. ${ }^{7}$ Further, the PVA fiber has high degree of crystallization as well. Consequently the PVA fiber gave the tensile strength as high as $16 \mathrm{~g} \mathrm{den}^{-1}$ It is worth mentioning that the in situ formed PVA fiber has a laminarly-associated fiber structure as shown in Figure 4. The length of the fiber was up to several centimeters.

\section{CONCLUDING REMARKS}

Spontaneous orientation and association of molecules have been reported only for the rigid chain polymers such as liquid-crystalline polymers. We report here that even the flexible chain polymer such as syndiotacticityrich PVA can produce spontaneous molecular alignment if the intermolecular attraction through polar pendant groups is strong enough to form phase-separation and maintain the shear-oriented structure. The in situ visualization of the saponifying system of syndiotacticityrich PVPi clearly showed that the spontaneous fiber formation was accomplished at the final stage of saponification by phase-separation, followed by shear-induced ori- 
entation of polymer molecules. Thus the in situ fiber formation process judged to include a conformational transition from flexible to rigid. The in situ fibrillation technology can be utilized in manufacturing the highly oriented and crystalline ultra thin PVA fibers. The importance of the in situ fibrillated PVA fiber lies in the similarity in structure with natural fibers with a large specific surface area and outstanding mechanical properties resulting from the perfect fiber structure. In addition, owing to the molecular ordering in the fiber the PVA fiber has a potential application for the precursors of active carbon fibers as well.

\section{REFERENCES}

1. W. S. Lyoo and W. S. Ha, Polymer, 37, 3121 (1996).

2. B. C. Kim, "Unspun Natural Pulp-Like Synthetic Fibers," in Polymeric Materials Encyclopedia, J. C. Salamone, Ed., CRC Press, New York, N.Y., 1996, pp 2350-2359.

3. W. S. Lyoo, B. C. Kim, and W. S. Ha, Polym. Eng. Sci., 37, 1259 (1997).

4. W. S. Lyoo, B. C. Kim, and W. S. Ha, Polym. J, 30, 424 (1998)

5. K. Yamaura, M. Maimoh, T. Tanigami, and S. Matsuzawa, J. Appl. Polym. Sci., 36, 1707 (1988).

6. N. Nakajima, Kobunshi Kagaku, 11, 142 (1954).

7. T. Yamamoto, S. Seki, R. Fukae, O. Sangen, and M. Kamachi, Polym. J., 27, 567 (1990).

8. W. S. Lyoo, J. Blackwell, and H. D. Ghim, Macromolecules, 31, 4253 (1998) 